\title{
A one year audit of the Colorectal Unit at Wits Donald Gordon Medical Centre: 2016-2017
}

\author{
DL Lutrin, ${ }^{1,2}$ HR Etheredge, ${ }^{3,4}$ GJ Oettle, ${ }^{1,2}$ N Harran, ${ }^{1,2}$ O Taback, ${ }^{1}$ N Lahoud, ${ }^{2}$ B Bebington ${ }^{1}$ \\ ${ }^{1}$ Colorectal Unit, Wits Donald Gordon Medical Centre, Johannesburg, South Africa \\ ${ }^{2}$ Department of Surgery, Faculty of Health Sciences, University of the Witwatersrand, South Africa \\ ${ }^{3}$ Wits Donald Gordon Medical Centre, Johannesburg, South Africa \\ ${ }^{4}$ Department of Internal Medicine, Faculty of Health Sciences, University of the Witwatersrand, Johannesburg, South Africa
}

Corresponding author: Dr Dean Lutrin (dean.lutrin@wits.ac.za)

\begin{abstract}
Background: Colorectal surgery has developed into an established surgical subspecialty in South Africa, however there is a paucity of data regarding the epidemiology and surgical outcomes of patients with colorectal disease in this country. The objective is to present the findings of a one-year audit of the Wits Donald Gordon Medical Centre (WDGMC) Colorectal Unit with specific reference to indications, surgical procedures and patient outcomes.

Methods: Patient files from December 2016 to November 2017 were included in a retrospective analysis. The MannWhitney U test was used to analyse continuous variables and the Chi-squared test was used to compare categorical variables. Results: During the audit period, 1264 patients were admitted to the Colorectal Unit and a further 564 outpatient endoscopic procedures were performed. There were 306 emergency admissions. 139 elective colorectal resections took place, with a $16 \%$ major complication rate, a $12 \%$ anastomotic leak rate and no deaths. Rectal resections constituted $66 \%$ of the operations and 34\% were colonic resections. The median length of stay for all patients undergoing resection was 9 days and there was no statistically significant difference in length of stay between open and laparoscopic cases.

Conclusion: The WDGMC Colorectal Unit manages a high volume of patients presenting with the full spectrum of colorectal disease.
\end{abstract}

S Afr J Surg 2019;57(3)

http://dx.doi.org/10.17159/2078-5151/2019/v57n3a2863

\section{Introduction}

Colorectal surgery has established itself as a distinct surgical subspecialty in South Africa (SA) and multiple dedicated colorectal units have been established. The current Certificate in Gastroenterology for Surgeons, of the Colleges of Medicine of South Africa, allows candidates to be examined in Colorectal Surgery as a subspecialty interest.

There is a paucity of data regarding the presentation and outcomes of patients with colorectal disease, particularly colorectal malignancy in SA. For example, the current guideline document for the management of non-metastatic colorectal malignancy as published by the Council for Medical Schemes of South Africa contains only two SA references, both of which relate only to patients with hereditary colorectal cancer syndromes. ${ }^{1}$ A paper has recently been published describing the epidemiology of colorectal cancer in a South African cohort of privately funded patients ${ }^{2}$ but this does not describe perioperative outcomes. Colorectal surgery comprises approximately $10 \%$ of general surgical operations but is responsible for approximately $25 \%$ of all surgical complications. ${ }^{3}$ We believe that there is a pressing need to quantify surgical practice and outcomes in SA.

Generating reliable South African data across the spectrum of colorectal surgery is essential. Data from Europe and the United States regarding scope of practice and surgical outcomes of patients treated in colorectal units is not easily extrapolated to the unique healthcare system of SA. The SA health system serves a different patient population and operates within a framework of socio-economic asymmetries, unique genetic heredity patterns and inconsistent access to care - even in the private sector where medical aid schemes often demand onerous co-payments for essential procedures.

This article provides a broad overview of the activity of a private academic colorectal surgical unit based on a one-year retrospective audit. The Wits Donald Gordon Medical Centre (WDGMC) Colorectal Unit (CRU) is a tertiary referral unit and manages a range of particularly complex cases. The CRU is one of the 'specialist and sub-specialist' teaching units of the Department of Surgery at the University of the Witwatersrand. Registrars in general surgery and subspecialty 
fellows in surgical gastroenterology rotate through the WDGMC CRU as part of their training.

\begin{abstract}
Aims
The main aim of this retrospective audit was to evaluate the activity of the WDGMC CRU over the period of a year, and make comparisons to international findings.

In this article parameters such as surgical indication, length of stay and postoperative complications for patients undergoing elective colorectal resections are emphasised. We hope that the information described in this paper contributes towards health policy decision making in the private sector in SA, particularly with reference to short term perioperative outcomes.
\end{abstract}

\section{Materials and Methods}

\section{Study setting}

The WDGMC CRU includes three full time colorectal surgeons and a part time endoscopist. The surgeons work in close multidisciplinary collaboration with physicians, gastroenterologists, intensivists, and other clinicians at the hospital. The CRU is supported by a number of allied healthcare practitioners including dieticians, stomatherapists, physiotherapists and psychologists. The CRU members participate in weekly oncology and monthly pelvic floor multidisciplinary team meetings.

\section{Study population}

Details of all patients admitted to the WDGMC CRU between December 2016 and November 2017 were consecutively captured onto an electronic research database (Airtable.com). Recorded variables included patient demographics, operative procedures, outcomes and complications. This information is routinely collected and used for internal audit as well as the unit's Morbidity and Mortality meetings. Patients under the age of 18 were excluded. The data was analysed in SPSS v24 and Tableau v10.5. The Mann-Whitney U test was used to analyse continuous variables and the Chi-squared test was used to compare categorical variables.

\section{Results}

Over the study period, 1264 eligible patients were admitted to the CRU and a further 564 patients were admitted as day cases for endoscopy. This equates to an average of 110 hospital admissions per month. There were 339 nonsurgical admissions during the audit period. Table 1 lists the top 10 admission ICD 10 codes for elective surgeries. Other admission diagnoses include hernias, abdominal pain, gastrointestinal bleeding, inflammatory bowel disease, and a wide spectrum of general surgical pathology. During the audit period there were 306 emergency admissions, 900 elective admissions, and 58 30-day readmissions. 925 operations were performed.
Table 1: Top 10 ICD 10 codes

\begin{tabular}{lc}
\hline Primary ICD 10 code (group) & $\begin{array}{c}\text { Percentage of Total } \\
\text { (Number of admissions) }\end{array}$ \\
\hline Rectal Cancer & $17 \%(154)$ \\
Perianal abscess \& fistula & $15.6 \%(140)$ \\
Colon Cancer & $8.1 \%(73)$ \\
Chronic anal fissure & $5.7 \%(51)$ \\
Rectal prolapse & $5.3 \%(48)$ \\
Haemorrhoids and related & $5.1 \%(46)$ \\
Faecal incontinence & $4.9 \% 44)$ \\
Benign colonic neoplasia & $3.4 \%(31)$ \\
Stenosis of anus and rectum & $1.8 \%(16)$ \\
Fistula of vagina to large intestine & $1.7 \%(15)$
\end{tabular}

An average of 77 surgical and 47 endoscopic procedures were performed monthly. Table 2 describes the top 10 operative procedures.

\begin{tabular}{lc} 
Table 2 - Top 10 procedures & \\
\hline Operation description & $\begin{array}{c}\text { Percentage of Total } \\
\text { (Number of cases) }\end{array}$ \\
\hline Fistula and abscess & $13.2 \%(125)$ \\
Closure of stoma & $6.3 \%(59)$ \\
Haemorrhoids (all procedures) & $6 \%(57)$ \\
Laparoscopic rectopexy (for prolapse) & $4.8 \%(45)$ \\
Laparoscopic colectomy & $4.3 \%(40)$ \\
Open proctectomy & $4 \%(38)$ \\
Botox injection for anal fissure & $3.9 \%(37)$ \\
Open colectomy & $2.8 \%(26)$ \\
Vascular access (ports) & $2.7 \%(25)$ \\
Transanal excision of rectal tumour & $2.6 \%(24)$
\end{tabular}

Colorectal resections are an important part of the practice of the WDGMC CRU. Over the study period, 139 elective resections were performed. The majority of operations were for malignant and premalignant conditions. A small number of operations for inflammatory bowel disease were undertaken $(\mathrm{n}=10,7 \%)$. The remainder of the resections were for conditions such as bowel obstructions, endometriosis, and fistulae (See Table 3). Of note, we perform very little elective surgical resection for diverticular disease $(n=3,2 \%)$.

\begin{tabular}{lc} 
Table 3 - Indications for resection & \\
\hline Indication for resection & Number of cases $\mathbf{n}=\mathbf{1 3 9}$ \\
\hline Neoplasia & $(107) 77 \%$ \\
Other & (20) $14 \%$ \\
Inflammatory bowel disease & (10) $7 \%$ \\
Diverticular disease & (3) $2 \%$
\end{tabular}

Rectal and distal sigmoid resections comprised $66 \%$ $(\mathrm{n}=92)$ of the colorectal resections and the remainder $(34 \%$ $\mathrm{n}=47$ ) were for colonic and small bowel disease. 
The majority (55\%) of patients undergoing colorectal resections had no complications (Clavien Dindo 0), 29\% had minor complications (Clavien Dindo $1 \& 2$ ) and $16 \%$ had major complications (Clavien Dindo $3 \& 4$ ). There were no deaths after elective resections during the study period (Clavien Dindo 5).

The overall anastomotic leak rate was $12 \%$ (11 leaks), all of which occurred in rectal procedures.

\begin{tabular}{lccc} 
Table 4 - Leak rate & & & \\
\hline Surgical Site & Leak & No Leak & Leak rate \\
\hline Colon \& terminal ileum & 0 & 47 & $0 \%$ \\
Rectosigmoid \& rectum & 11 & 81 & $12 \%$
\end{tabular}

The median length of stay for all colorectal resections was 9 days (interquartile range 7-16 days). The median length of stay for patients undergoing terminal ileal and colonic surgery was 8 days (IQR 6-13) and the median length of stay for patients undergoing rectal/rectosigmoid resections was 11 days (IQR 7-16), but this difference was not statistically significant $(p=0.07)$

There was no significant difference in the overall length of stay between open and laparoscopic surgery $(p=0.09)$ and there was no difference between the groups of colonic $(\mathrm{p}=0.65)$ and rectal $(\mathrm{p}=0.09)$ surgery when looking at these separately. The major complication rate was $11 \%$ for laparoscopic surgery and $20 \%$ for open surgery but this difference was also not statistically significant $(\mathrm{p}=0.09)$.

\section{Table 5 - Length of stay (days)}

\begin{tabular}{lccl}
\hline Surgical Site & Open & Laparoscopic & \\
\hline Colon \& terminal ileum & 10 & 7.5 & $\mathrm{p}=0.65$ \\
Rectosigmoid \& rectum & 11 & 10 & $\mathrm{p}=0.09$ \\
Overall & 10.5 & 8 & $\mathrm{p}=0.09$
\end{tabular}

For patients undergoing elective colorectal resections, the rate of paralytic ileus was $13.7 \%$ and the rate of superficial surgical site infection was $12.9 \%$.

The 30-day readmission rate for all patients was $2 \%$ and it was $0.7 \%$ for patients undergoing resections.

\section{Discussion}

The top 10 admission diagnoses and the top 10 procedure types provide a good overview of surgical procedures performed in the WDGMC CRU. The ratio of rectal and distal sigmoid resections to colonic and small bowel resections reflects the nature of the CRU as a tertiary referral unit undertaking more complex cases. Rectal and distal sigmoid resections have been grouped together in order to more simply compare this group with the group of patients undergoing pure colonic surgery. Additionally, the height of the anastomosis is not specifically captured in our database. We do not have data from other units to allow for comparison, but we feel that the spectrum of pathology managed by the CRU is typical for an academic colorectal unit managing a complex caseload.

A rather small number of the elective colectomies are performed for diverticulitis in the WDGMC CRU. There is evidence of an increasing number of elective colectomies being performed for diverticulitis ${ }^{4}$ but this may be contrary to the guidance provided by current evidence ${ }^{5}$ that most patients do not require surgery.

WDGMC does not have an emergency unit attached to it. This may account for fewer colorectal emergencies such as acute large bowel obstructions and perforated diverticulitis being managed in the unit.

\section{Length of stay}

Length of stay in hospital is often used as a surrogate for quality of care; but length of stay targets in Europe and the United States should be extrapolated to the SA healthcare environment with caution. In a UK National Health Service (NHS) audit of colorectal malignancy, the median length of stay for all colorectal surgery was 7 days with a $10 \% 30$-day readmission rate. ${ }^{6}$ The median length of stay in our unit was 9 days with a very low readmission rate. This finding suggests that a longer stay in hospital may result in fewer readmissions. It is hypothesised that patients in our hospital stay longer because of the lack of suitable outpatient support available in SA, but our readmission rate is very low when compared to data from the NHS. It is possible that longer length of stay in hospital may equate to a cost-saving mechanism for healthcare funders in SA, who are not required to fund the management of readmissions to the extent that this is done in other countries.

Minimally invasive surgery has been advocated as a tool to improve short term outcomes in colorectal surgery. It is interesting to note that in this series there was no statistically significant difference in median length of stay ( 9 days for open and 7 days for laparoscopic) and the rate of major complications ( $20 \%$ for open and $11 \%$ for laparoscopic) post colorectal resection between those undergoing open vs laparoscopic surgery. This could be due to the small number of cases included in the analysis. Laparoscopic surgery appears to have significant clinical benefits to patients by reducing length of stay and complications and we are auditing the results of the WDGMC CRU on an ongoing basis to determine whether this is the case in our setting.

\section{Surgical complications and readmissions}

In the CRU we use the Clavien Dindo classification system to record our 30-day complication rate. The Clavien Dindo classification system is a method of categorising complications based on how the complication is managed. ${ }^{7}$ Worldwide, it has become the standard tool to quantify surgical complications. Grade 1 and 2 are considered minor complications and include wound infections, postoperative ileus, and pneumonia. Grade 3 complications require an intervention such as a reoperation or percutaneous drain. Grade 4 complications are indicated by complication-associated single or multi-organ failure. Grade 5 complications are deaths. In this study, $16 \%$ of patients undergoing an elective colorectal resection had a 
major complication and $29 \%$ had a minor complication. For comparison, a study from the VU University Medical Centre in Amsterdam demonstrated that $20 \%$ of patients undergoing major gastrointestinal surgery had major complications and $15 \%$ of patients had minor complications. ${ }^{8}$ The complication rates reported in this study appear to be consistent with international registry-based reports and may suggest that the quality of surgical skill and care provided by the WDGMC CRU is of international standard. ${ }^{9}$

The majority of patients undergoing major colorectal resections are assessed by either a geriatrician or intensivist preoperatively and operations are often scheduled to allow for a period of prehabilitation - a well defined program to optimise the patient's physiological condition prior to surgery. ${ }^{10}$ It is believed that a formal Enhanced Recovery After Surgery (ERAS) programme helps reduce complications and postoperative length of stay. ${ }^{11}$ Our hospital does not yet have a formal ERAS programme due to both cost and logistical constraints, but we are actively implementing postoperative strategies consistent with ERAS principles. These strategies, as well as our relatively longer length of stay, may further explain our low readmission rate.

In our unit, postoperative ileus is a major obstacle to early discharge. Excessive postoperative intravenous fluid and opiate usage increase the rate of paralytic ileus. ${ }^{12,13}$ Looking beyond the audit period of this report, formal strategies to limit postoperative fluid and opiate usage appear to be effective with respect to lowering the ileus rate.

The leak rate for rectal resections in this study was $12 \%$. The average leak rate for rectal resections is quoted as being approximately $10 \% .{ }^{14}$ Whilst there were no anastomotic leaks in colonic operations during the study period, the rate in this unit for these leaks is not zero. Rectal and distal sigmoid resections comprised two thirds of the colorectal resections performed in the CRU. In other audits of colorectal cancer, approximately one quarter of the resections are for rectal disease. The ratio of colonic to rectal resections in this audit is most likely a reflection of it being a referral unit.

\section{Multidisciplinary Team Meetings}

All patients presenting to the CRU with colorectal cancer are discussed at a weekly multidisciplinary gastrointestinal oncology meeting (MDT). This meeting is attended by approximately 20 individuals from surgery, radiation oncology, medical oncology, radiology and pathology. The utility of the MDT in clinical oncology has been well verified and is rapidly becoming mandatory around the world. ${ }^{15}$ Setting up an MDT in the private sector has been challenging as there is no obligation to attend the meeting and participation relies on the 'goodwill' of attendees. We feel that the continued success of the meeting is a result of the value that participants attach to it, as it provides a platform to optimise patient care. This is especially pertinent in the WDGMC CRU, where many colorectal cancer cases are unusually complicated.

\section{Conclusion}

This audit demonstrates that the WDGMC CRU has a case mix and case volume that adds useful information to our understanding of colorectal disease in South Africa. We believe that this study will contribute to defining the epidemiology and outcomes of colorectal disease in this country. Metrics such as length of stay and complication rates are important for planning resource availability and justifying funding for certain procedures and longer hospital stays. It is hoped that the results of this study will help develop colorectal surgery in South Africa.

\section{Recommendation}

Formal ERAS programmes have been well validated as a method to reduce both length-of-stay and postoperative complications. We believe that there would be value in setting up a formal ERAS program within the WDGMC CRU. These programs can be costly to implement but could potentially save money overall. We believe that both the medical aid industry and private hospital groups have an important role here.

Shorter hospital stays at the expense of higher readmission rates are not in our patients' best interests. Mandatory preoperative consultation with a physician or geriatrician may be a valuable way of reducing postoperative complications. ${ }^{16}$ The use of a colorectal cancer MDT is helpful and should become the standard of care for all gastrointestinal oncology.

\section{Ethics Approval}

The study was approved by the Wits Human Research Committee (medical clearance \#M140645 and M104841).

\section{Acknowledgments}

We would like to thank the research office at the Wits Donald Gordon Medical Centre for their assistance. Petra Gaylard from Data Management and Statistical Analysis, Johannesburg, South Africa provided statistical advice.

\section{Author declarations}

The authors confirm that all authors have made substantial contributions to the article.

The authors confirm that the work submitted is original and does not transgress the plagiarism policy of the journal.

\section{Conflict of interest statement}

None of the authors has a conflict of interest to declare.

\section{Funding sources}

Wits Donald Gordon Medical Centre Research Funds

\section{REFERENCES}

1. Maramba E. Draft PMB definition for early stage colon and rectal cancer [Internet]. http://www.medicalschemes.com/ files/PMBDefinitionProjectDrftBnftDfntnErlyStgClnNRctl. pdf. 2017 [cited 2018 Jun 6];1-19. Available from: http://www. medicalschemes.com/files/PMB\%20Definition\%20Project/ DrftBnftDfntn_ErlyStgClnNRctl_20170303.pdf

2. Brand M, Gaylard P, Ramos J. Colorectal cancer in South 
Africa: An assessment of disease presentation, treatment pathways and 5-year survival. S Afr Med J. 2018;108(2):118-5. doi: 10.7196/SAMJ.2017.v108i2.12338

3. Schilling PL, Dimick JB, Birkmeyer JD. Prioritizing Quality Improvement in General Surgery. J Am Coll Surg. 2008;207(5):698-704. doi: 10.1016/j.jamcollsurg.2008.06.138

4. Etzioni DA, Mack TM, Beart RW Jr., Kaiser AM. Diverticulitis in the United States: 1998-2005. Ann Surg. 2009;249(2):210-7. doi: 10.1097/SLA.0b013e3181952888

5. Feingold D, Steele SR, Lee S, et al. Practice Parameters for the Treatment of Sigmoid Diverticulitis. Dis Colon Rectum. 2014;57(3):284-94. doi: 10.1097/DCR.0000000000000075

6. Boyle J, et al. National Bowel Cancer Audit Annual Report 2017 Version 2 [Internet]. Available from: https://www.nboca. org.uk/content/uploads/2017/12/NBOCA-annual-report2017-v2.pdf [Accessed 2019 June 18].

7. Dindo D, Demartines N, Clavien P-A. Classification of Surgical Complications. Ann Surg. 2004;240(2):205-13. doi: 10.1097/01. sla.0000133083.54934.ae

8. Straatman J, Cuesta MA, de Lange-de Klerk ESM, van der Peet DL. Hospital Cost-Analysis of Complications after Major Abdominal Surgery. Dig Sur. 2015;32(2):150-6. doi: $10.1159 / 000371861$

9. Lee L, Liberman $\mathrm{S}$, Charlebois $\mathrm{P}$, et al. The impact of complications after elective colorectal resection within an enhanced recovery pathway. Tech Coloproctol. 2018;22(3):1-9. doi: 10.1007/s10151-018-1761-x
10. Jankowski CJ. Preparing the Patient for Enhanced Recovery After Surgery. Int Anesthesiol Clin. 2017;55(4):12-20. doi: 10.1097/AIA.0000000000000157

11. Ljungqvist O, Scott M, Fearon KC. Enhanced Recovery After Surgery. JAMA Surg. 2017;152(3):292-7. doi: 10.14797/mdcj$14-2-77$

12. Chapman SJ, Pericleous A, Downey C, Jayne DG. Postoperative ileus following major colorectal surgery. Br J Surg. 2018;15(10):485-14. doi: 10.1002/bjs.10781

13. Basse L, Jakobsen DH, Billesbølle P, Werner M, Kehlet H. A clinical pathway to accelerate recovery after colonic resection. Ann Surg. 2000;232(1): 51 - 57. doi: 10.1097/00000658200007000-00008

14. Paun BC, Cassie S, MacLean AR, Dixon E, Buie WD. Postoperative Complications Following Surgery for Rectal Cancer. Ann Surg. 2010;251(5):807-18. doi: 10.1097/ SLA.0b013e3181dae4ed

15. Soukup T, Lamb B, Arora S, Darzi A, Sevdalis N, Green J. Successful strategies in implementing a multidisciplinary team working in the care of patients with cancer: an overview and synthesis of the available literature. JMDH 2018;11:49-61. doi: 10.2147/JMDH.S117945

16. Leeds IL, Canner JK, Gani F, et al. Increased healthcare utilization for medical comorbidities prior to surgery improves postoperative outcomes. Ann Surg. 2018;1-8. doi: 10.1097/ SLA.0000000000002851 